\title{
A Qualitative Inquiry: Pilot Testing of Qualitative Research Methodology and NVivo in the Field of International Academic Collaboration Between HEIs
}

\author{
Peter Waring Lei, Mahdokht Sedaghat \\ International Business Academy, Kolding, Denmark
}

\begin{abstract}
The proposed study has the potential of enhancing successful higher education (HE) management and decision-making processes by increased awareness of international academic collaboration (IAC) influences, thus acting as an "aide-memoir" of issues to HE management. The purpose of this pilot study is to test the appropriateness of research questions, methodology, methods, and techniques applied. Concepts and themes emerging should form the basis for future research of the IAC phenomenon. The paper provides a critical evaluation of the research paradigmatic stance. Justification of methodology and measures of trustworthiness, such as transparency in the research process, decisions, and procedures, provided using reflective research workbook unfolding procedures and decisions in the research process. Data were collected through semi-structured interviews among participants selected on basis of their affiliation with HE different organisational levels. Theoretical framework and key concepts were identified through critical literature review. Data analysis was assisted using NVivo software. NVivo was used to code data and various cluster and pattern analysis. Initial coding and advanced coding were based on identified theoretical framework, reflective workbook memos transcripts, and node properties and transcripts of participant interviews. Concepts and themes emerging from the data analysis were further interpreted using analysis of narrative and classified into meta-categories, e.g., higher order nodes. A conceptual model of successful IAC was identified and reported in the conclusion part, reflecting back upon the research questions including an evaluation of data saturation and the limited scope of the pilot study.
\end{abstract}

Keywords: international academic collaboration (IAC), higher education (HE), NVivo, Grounded Theory

\section{Introduction}

The overall aim of the proposed paper is to develop a conceptual framework for evaluating international academic collaboration (IAC) and to identify a conceptual model for successful IAC within a UK-DK context.

Ideally, the conceptual framework would contribute to successful management and increased internationalisation of higher education (HE).

The paper provides a critical evaluation of philosophical, epistemological, and ontological considerations including ethical considerations. Transparency in the research process, decisions, and procedures is provided through a comprehensive reflective diary by describing the foundations of the analytical process, the reasoning, 
and a continuing critical discussion.

A comprehensive reflective research workbook was acting as a research memo. It helped delivering transparency in the research process in terms of philosophical and methodological stance, data collection process, coding and analysis of data, interpretation of data, identification of emerging concepts, and synthesis. The reflective research workbook was entered into NVivo as an external source.

The paper will explain findings with reference to published literature and the applied research framework. Findings reflected back to the research questions. Synthesis of research findings is reported and presented as a conceptual model of successful IAC.

\section{Research Framework}

The research philosophy could significantly influence the role of theory (Kozlowski, Chao, Grand, Michael, Braun, \& Kuljanin, 2013) in research (Carter \& Little, 2007), and Burrell and Morgan (1979) defined four paradigms in business and management research (Bryman \& Bell, 2011). The necessity of interpretative orientation is imminent when researching novel phenomena (Eisenhardt, 1989). The positivist and objectivist orientation is less adequate within unexplored and novel research areas due to their theory dependency and theory lock-in. Researchers must remain open-minded and creative (Janesick, 2001) towards emergence of novel concepts. In this paper, the latter is achieved by choosing an interpretative paradigmatic stance committed to the constructionist ontological orientation, hence deemed appropriate in this pilot study of IAC phenomenon.

The epistemological perspective is important, because it means the overarching structure of the research including the nature of evidence gathered, from where it is gathered and how the data are interpreted (Easterby-Smith, Thorpe, \& Lowe, 2002).

The phenomenon of IAC observed as an evolving reality subject to constant change and members of staff is an integral part of the studied phenomenon. This study does not adhere to determinism, because it would ignore that members of the organisation are influencing and changing the organisation constantly. This study rests upon the assumption that IAC is shaped by the organisational setting and individual members of the organisation involved in the collaborative process (Burrell \& Morgan, 1979).

Among researchers, there are diverse viewpoints on how paradigmatic positions are affecting and constraining research methods. Choice of research paradigm should not rigidly lock research into one particular type of either pure qualitative or pure quantitative and the distinction is not always perfect (Bryman \& Bell, 2011). Although research approaches are generally divided into either inductive or deductive, often both methodologies are applied within the same research (Robertson, Mullan, \& Todd, 2014). Knox (2004) argued that philosophical consistency is necessary while methodological pluralism is acceptable.

The epistemological orientation is constructivism and the research has an emphasis on understanding of the processes rather than providing an explanation to them. The findings are subjective because other researchers would arrive at different conclusions using the same data sets.

The latter question about replicability gives rise to discussion of measures of accuracy and quality in qualitative research. It is of essence for the quality of the research that transparency is established through detailed description of the decisions and processes of the data analysis. With a high degree of transparency, it should be possible for other researchers to replicate the study although such studies most likely would arrive at different findings due to subjective interpretation of data. This paper has emphasis on ensuring validity through 
a high degree of transparency in the research process, i.e., decisions made and procedures for coding, categories and concepts by describing, and entering these into the reflective workbook (Zhang \& Wildemuth, 2009). Presumably all researchers strive towards highest possible quality in their research, whether quantitative or qualitative. Some researchers argue that quality in qualitative research is measured by consistency between epistemology, methodology, and method (see Figure 1). Others argue that generalisation of knowledge created is a measure of quality (Flyvbjerg, 2006). Further, in a research paper, Stacy, Carter, and Little (2007) argued that consistency is less important than the authors' eight universal criteria for evaluation of qualitative goodness. The author is exploring and identifying eight key markers of quality in qualitative research including worthy topic, rich rigor, sincerity, credibility, resonance, significant contribution, ethics, and meaningful coherence.

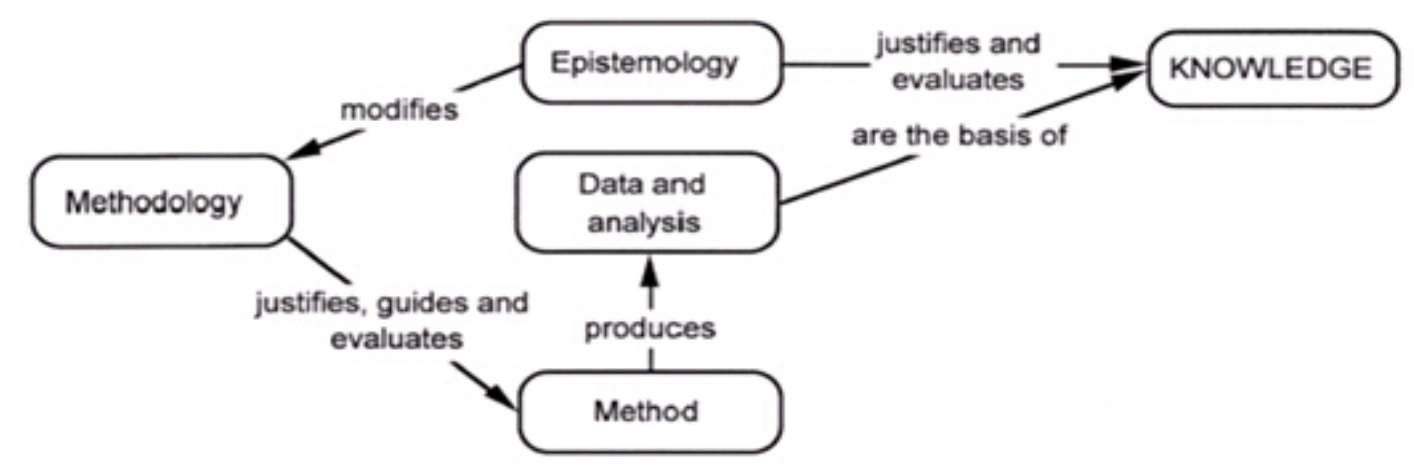

Figure 1. The simple relationship between epistemology, methodology, and method (Carter \& Little, 2007).

Another aspect of importance is the role of theory in research, whether a qualitative or a quantitative strategy is applied. In this inductive research paper, the role of theory is less deterministic since the basis of the research is the empirical orientation from which often new perspectives of the phenomenon is developed, i.e., the link to reality (empirical) is stronger than in deductive research. Findings are thus context-specific (Eisenhardt, 1989), which increases the relevance of the findings as each IAC has its specific setting in terms of organisation and members.

A modified analysis of narratives is applied herein (Polkinhorne, 2005), because this would enhance identification of coding aspects and produce general knowledge concepts. The initial coding in this paper is mainly inductive driven rather than theoretical (Bailey \& Jackson, 2003). Concepts and codes are explored using content analysis that goes beyond merely objective word count and content of the nodes in order to infer meanings and themes (Zhang \& Wildemuth, 2009). The authors are describing qualitative content analysis, inductive development of categories, and deductive application of categories.

Intuition and creativity is applied throughout this research as this would enhance inquisitive probing of identified concepts, inductive reasoning, and inferences of meanings (Janesick, 2001).

Qualitative data collection methods included in this study comprise interviews. Data management methods include audio-recording, transcription, transcript checking, and the use of computer-assisted analysis software (Nvivo). Data analysis methods include constant comparison, memo writing, and concept theory building (Charmaz, 2006; Glaser \& Strauss, 1967).

The paper is offering a qualitative inquiry based on Grounded Theory methodology and different analytical techniques identified within Grounded Theory (Glaser \& Strauss, 1967). An inductive approach uses qualitative methodology, methods, and techniques, such as semi-structured interviews, reflective workbook 
with memos, inductive concept development, content analysis, analysis of narrative, acknowledging creativity and intuition in the research process, purposive sampling, initial and advanced coding, categorisation, emergence theory (Kozlowski et al., 2013), concept development, and triangulation.

\section{Literature Review}

The shift from elite to mass together with marketization of HE has led to severe competition (Sporn, 1999), and globalization has also led to more and more strategic alliances among multiple partners across national borders (Chan, 2004). With the increasing commercialization, HE management is facing new strategic options, such as internationalisation in terms of international strategic alliances (ISA), which could include collaborative partnerships between higher education institutions (HEIs) or HE and industry.

It is evident that the paradigmatic position of the researcher whether functionalist aligned with positivism and objectivism (Nielsen, 2003; Ayoubi \& Massoud, 2007) or interpretative aligned with constructionism (Vauterin, Linnanen, \& Marttila, 2012; Li, Roberts, Yan, \& Tan, 2014) significantly influences the research method and methodology.

The research by Nielsen (2003) illustrated that a quantitative research using exploratory factor analysis was able to explore novel correlations by developing five underlying determinants of ISA (Nielsen, 2003). Although the topic was not IAC, but ISA, the findings are of significant interest because they are concerned with main drivers of ISA. The researcher concluded that ISA partner choice is a function of strategic motivation and varies significantly with governance mode and partner nationality, proposing that further in-depth research was needed for the development of a holistic understanding of how partnering for ISA experienced and to devise methods for successful partnering behavior. In the researcher's view, this suggests that nationality and management attitude are important determinants in the process of IAC.

In a qualitative inquiry (Ayoubi \& Massoud, 2007) using coding techniques, 74\% of UK universities' mission statements include international dimensions. In addition, $48 \%$ of these universities are internationally active and pursuing IAC HE, suggesting that there is a significant potential for IAC, which has not yet been implemented.

In a third research paper on academic-industry collaboration, the researchers concluded that the conceptualization of the international academia-industry collaborative partnerships needs to be extended to incorporate elements concerning the power, impact, and management of the boundary roles (Vauterin et al., 2012). Thus, suggesting that concepts of reciprocity, power balance between partners, and resource dedication to operation of the collaborative component are important determinants for successful collaboration.

In a more recent study, Li et al. (2014) found that the scale of academic and organizational knowledge sharing was affected by knowledge attributes and collaborate characteristics. While knowledge sharing in China-UK HE alliances displays numerous similarities with that occurring in other industries, this research paper reveals features that are distinct to IAC. Knowledge sharing presumed to be an important aspect of IAC, which needs to be included into future research on ISA.

The literature review suggests that research of international collaborative partnerships and ISA within industry is plenty, although it seems that the specific field of IAC is under researched as it was only possible to identify a few recent and relevant academic research papers addressing the particularities of IAC HE. Theory and conceptual frameworks of ISA were well described in academic literature. Theories are all substantive and middle range theories and focus on the factors determining partner choice and drivers of ISA. 
The research reviewed herein illustrates that both qualitative and quantitative research approaches apply to the phenomena. The literature review further suggests that interpretative research paradigm could lead to discovery of new relationships between factors and determinants of successful ISA.

\section{Conceptual Framework and Definition of Research Question}

Concepts identified through the review of previous research are the following: internationalization of HE, strategic fit/match, power relations, institutional and regulatory aspects, organisational culture, diffusion of teaching, convergence/divergence, knowledge sharing, innovation, communication, organisational learning, student learning experience, and cost/benefits.

The below research aspects are derived from the identified concepts complemented by the researcher's own observations of the phenomenon of IAC and further refined through the Delphi method.

The research question identified is: Why are some IAC HEIs successful? The different aspects of the research question are strategic fit/match, institutional and regulatory aspects, and organisational culture.

It could prove difficult to establish why some IAC HEIs are successful and efficient. The research of such phenomenon therefore requires certain specificity as to how, what, when, and who. The research question designed to capture the most essential aspects of the phenomenon of IAC without being indefinite and too broadly worded.

Other critically important issues are the causal relationships between the different aspects and concepts. As sufficient data and knowledge have not yet been obtained, it would be appropriate to inform the research questions by empirical knowledge from the earlier literature review and the case studies therein.

\section{Data Collection}

The selection of interview participants was determined by the request for rich and varied data from members of HEIs having experienced IAC, additional interview participants must be distributed across top, mid, and low organisational level. At this initial stage of the pilot study, the research topic is assumed to be better informed by interviewing selected respondents, hence a purposive sampling technique is selected (Hutchison, Johnston, \& Breckon, 2010). Polkinhorne (2005) suggested multiple participants in order to deepen the understanding of the investigated experience. Access was properly negotiated with gate keepers. Three interview participants were invited by Invitation Letters explaining the study and why they had been selected to participate, Attached Information Sheets, and Written Consent Forms. During the entire data collection and research process, ethics codes were observed.

To minimise the degree of intrusion and other influences, the research asked the participants to conduct the interviews in their respective offices. Interviewed outside their comfort zone, the participants are more likely to be influenced by anxiety, etc., and would maybe not answer as detailed and natural. The researcher wanted them to answer openly, honestly, and as detailed as possible to the research question.

The interviews were audio-recorded and memos were written during the interviews. The audio-recorded interviews were transcribed and entered into NVivo.

\section{Data Analysis and Discussion}

The data were analyzed using NVivo. Nodes representing concepts were also identified using NVivo. The emergent concepts were further developed and analyzed using reflective research workbook. Ongoing conceptual 
development, sampling, and initial and advanced coding were made in an iterative process of analysis and decisions were recorded in the research workbook. Coding of nodes and interpretation of meaning of text and narratives were facilitated by applying Polkinhorne's analysis of narrative in a moderated version, which is associated with the logical approach of Strauss and Corbin's coding (Bailey \& Jackson, 2003), supposing a pure open-minded approach would result in a true account of IAC and aspects researched. The objective of the analysis herein is to produce knowledge of general concepts that look across several people and situations hence searches for commonalities. Determinants of narrative are prior knowledge, preconceived notions (see Figure 2), research questions, and interview themes. These will influence the coding and analysis of data.

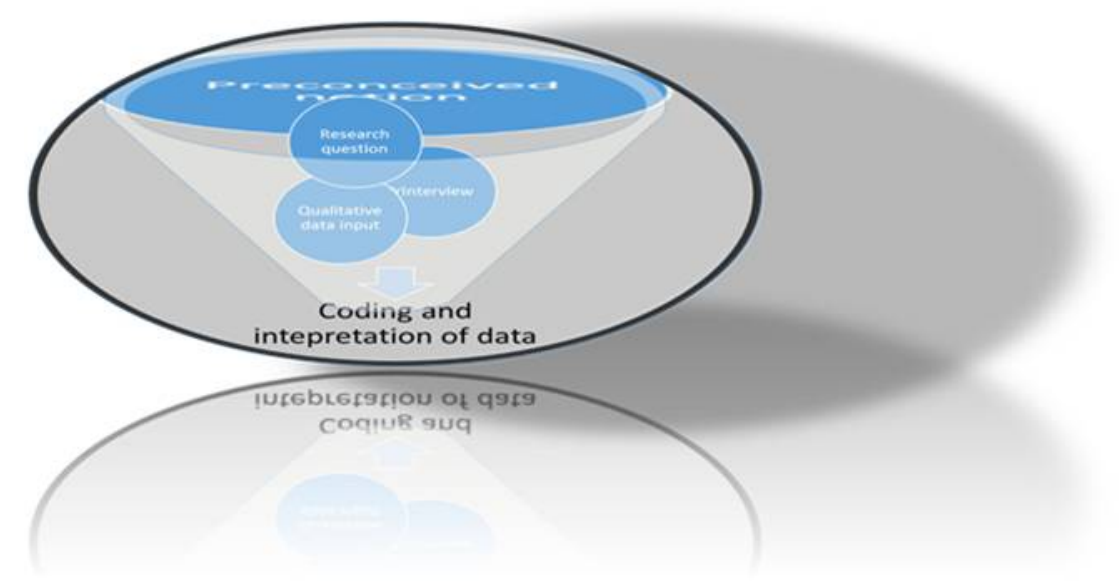

Figure 2. Preconceived notions by the author (Lei, Peter Waring, 2014).

Initially, the following key concepts (nodes) were defined: strategy and institutional, regulatory, and organizational culture. The transcribed text was organized into nodes using auto-coding. Each question in the semi-structured interview formed a node and the answers from the three respondents formed the code. A word frequency query was run in NVivo and the result was analyzed. The 100 most frequent words were scrutinized and from the list, 19 relevant concepts were identified by comparing words to the meaning and context of the transcripts. The 19 concepts were entered into NVivo as nodes and coded. From this process, new concepts emerged.

The new concepts analyzed using in-depth coding by establishing relationship between nodes and sources, nodes, building parent nodes with child nodes and code. Ninety-six new nodes were identified and coded. Nodes were systemized into hierarchy by using child nodes thus establishing relationships related to each other by child nodes. Often, coding the same text under two or more nodes where several relationships identified, ex. the concept of trust relates to other concepts, ex. perceived successful IAC, (actual) successful IAC, quality assurance, and personal relationship.

Further analysis includes comparison of concepts through matrix and other visual queries involving the 96 new nodes developed. Figure 3 and 4 are abstracts from NVivo showing a range of the concepts developed.

The open-minded coding and analysis process allowed many new concepts to emerge. The data need to organized into meta-categories, thus analysis of the developed coding was conducted using different cluster analysis. Nodes were clustered by coding similarity and nodes clustered by wording similarity. Analysis of coding similarity clusters reveal relationships between concepts such as trust/reciprocity and attitude/flexibility and adaptability. This indicates that partner confidence at a personal, organizational, and strategic level 
enhanced by communication, social interaction, and common culture relates to perception of mutual beneficial partnerships. Very illustrative of the importance of trust is the following narrative extracted from coding of trust/negative case:

I work as external examiner for a UK university. They have collaboration with Kuala Lumpur. I perceive and I observe their collaboration - put this on an outside track — and the trust there is a lot less than I perceive here. I think there is a distance factor that affects trust that is a cultural, culture with a large $\mathrm{C}$ and a human culture, cultural differences. They are not European cultures the Asian cultures that create, I think, a little bit of mistrust. I also observed that there is a dominant approach by the UK university over their partners in Malaysia. They have to micro-manage the collaboration, which is part of the mistrust. We at London South Bank University never micro-manage, we let a collaborative partner lead when necessary.

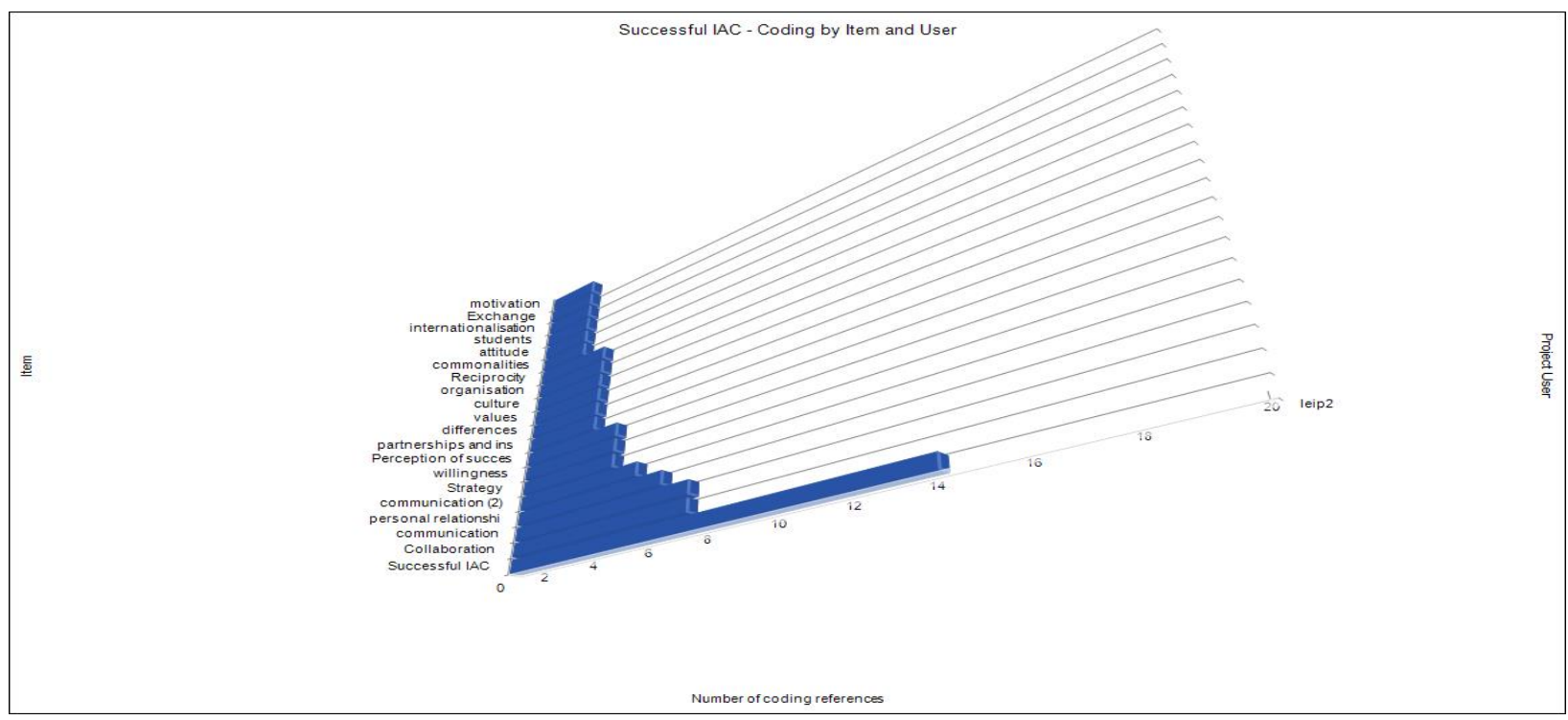

Figure 3. An extract from NVivo by the author (Lei, Peter Waring, 2014).

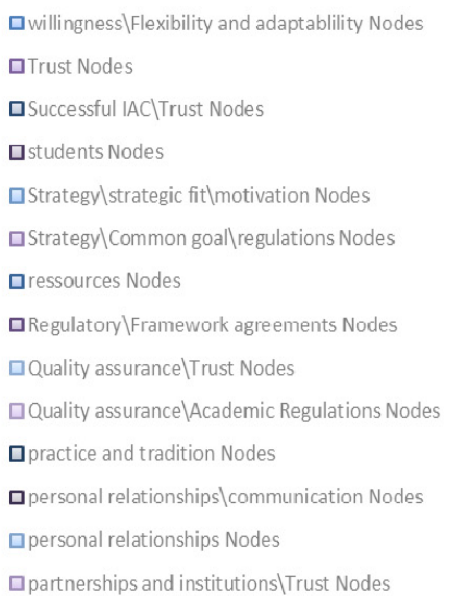

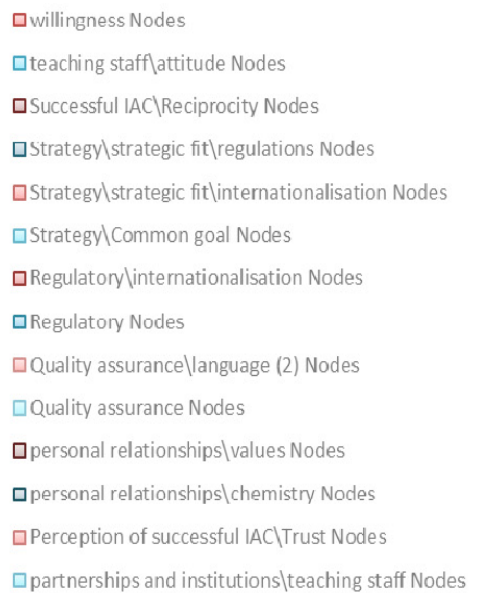

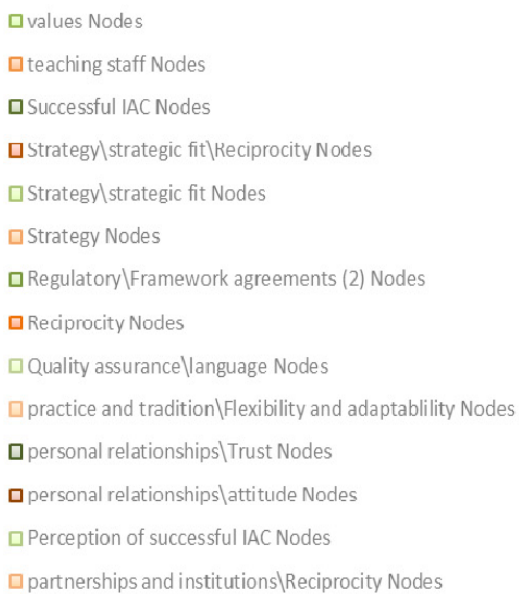

Figure 4. Another extract from NVivo by the author (Lei, Peter Waring, 2014).

The above quote interpreted as evidence of impact of non-trust and illustrates the negative case of the concept of trust.

Further analysis and comparison with theoretical framework identified earlier nodes, which are categorized into three higher order categories: Basic factors classified as determinants prerequisite of entering 
into collaborative agreements between HEIs, such as a framework agreement, skilled staff, compatibility of systems (IT), etc.. Maintenance factors classified as determinants necessary to operationalize and maintain the collaboration beyond the basic level such as appropriate resource allocation, communication, etc.. Advanced factors classified as determinants, which would further the alliance and provide for successful collaboration: flexibility and adaptability, openness, reciprocity, and trust.

Cluster analysis indicates that clusters of nodes with same wording connected by more than pure wording similarity, namely in terms of overlapping coding. Trust clustered with flexibility and adaptability. Comparing this finding with meta-category of advanced factors, it becomes clear that the concept of trust, reciprocity, openness, flexibility, and adaptability classified as determinants, which would improve the collaboration and provide for successful IAC.

\section{Conclusion}

Successful IAC is based upon a variety of determinants, but the data analysis from the pilot study research suggests that there are different stages in the process of collaboration spanning across basic requirement over maintenance of the collaboration to more advanced stages where specific identified factors prompt successful IAC.

The findings are corresponding with the preconceived notion although the concepts of reciprocity and trust are not explicitly identified through the literature review. Trust and reciprocity are underlying implicit determinants of smooth collaboration, hence the narrative of negative case above. Their perception of mutual beneficial relationship inherent in reciprocity was not directly anticipated when defining the research questions but became apparent during the analysis of data, similar with trust.

A “conceptual model for successful IAC HE” emerges from analysis of data (see Figure 5).

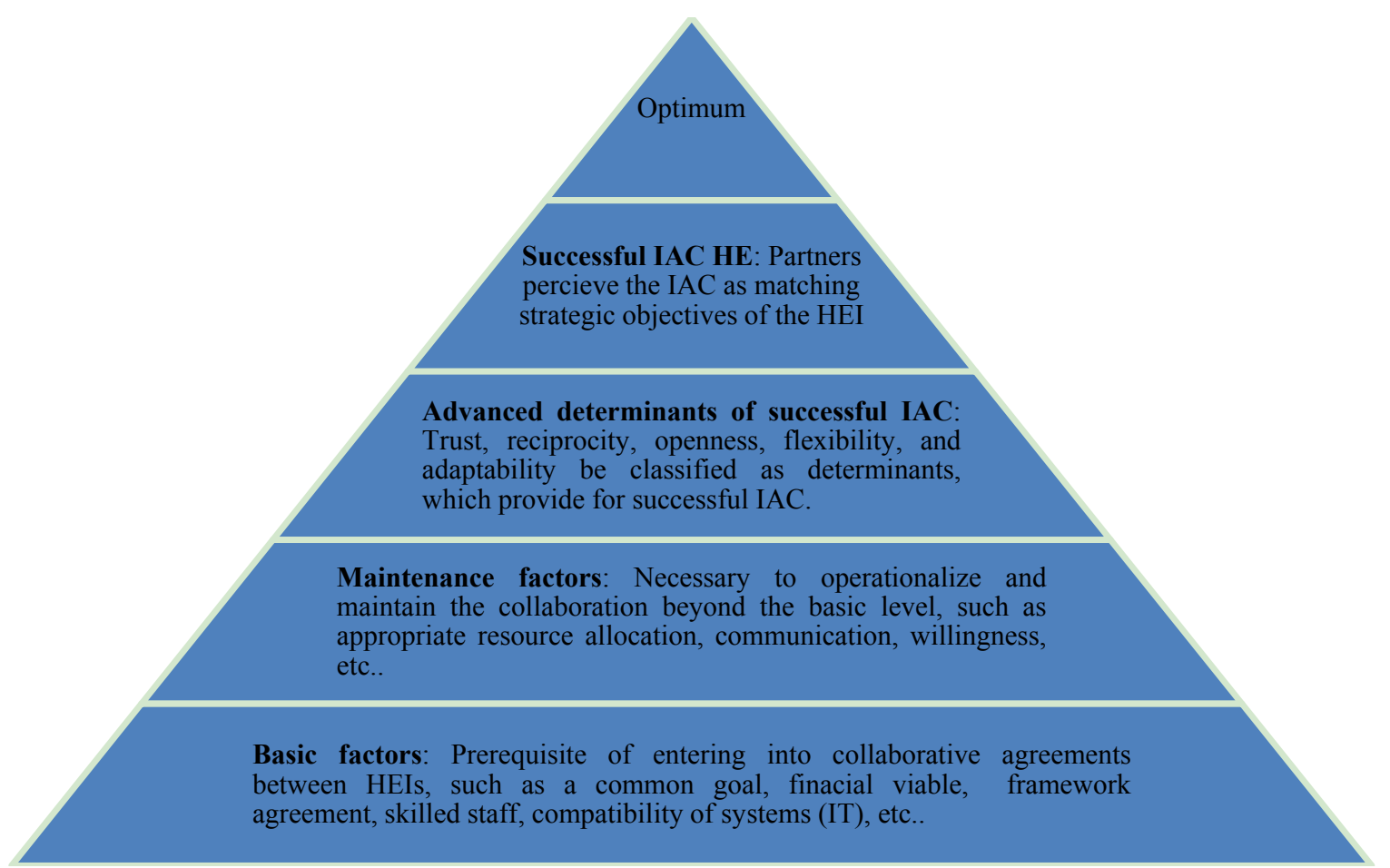

Figure 5. Conceptual model of successful IAC HE by the author (Lei, Peter Waring, 2014). 


\section{Limitations}

The sample is not representative for all DK-UK HEI IAC due to the narrow sample size, i.e., the actual data saturation point was not reached.

This pilot study has neither achieved data saturation nor saturation in terms of conceptual development from data analysis. This is due to formal constraints, such as word count and maximum allowed length of the paper.

This inductive study could be disapproved for being very subjective compared to a possible qualitative study of the same phenomenon.

\section{Future Research}

The proposed model should be further researched and developed by future qualitative inquiry or quantitative research project.

In a future research project, the sample size should be expanded to include at least three UK-DK HEI IAC and should comprise different parts of the HEI staff. For example, interviews with staff from the international offices would shed light on technical and formal aspects.

A different approach study could be successfully carried out, i.e., a quantitative study. The challenge is probably to establish a relevant theoretical framework in order to construct hypotheses to test.

\section{References}

Ayoubi, R., \& Massoud, H. K. (2007). The strategy of internationalization in universities: A quantitative evaluation of the intent and implementation in UK universities. International Journal of Educational Management, 21(4), 329-349.

Bailey, D. M., \& Jackson, J. M. (2003). Qualitative data analysis: Challenges and dilemmas related to theory and method. American Journal of Occupational Therapy, 57(1), 57-65.

Bazeley, P. (2009). Analysing qualitative data: More than identifying themes. Malaysian Journal of Qualitative Research, 2 , 6-22.

Bryman, A., \& Bell, E. (2011). Business research methods (3rd ed.). Oxford, U.K.: Oxford University Press.

Burrell, G., \& Morgan, G. (1979). Sociological paradigms and organisational analysis. Hants: Ashgate.

Carter, S. M., \& Little, M. (2007). Justifying knowledge, justifying method, taking action: Epistemologies, methodologies, and methods in qualitative research. Qualitative Health Research, 17(10), 1316-1328.

Chan, W. Y. (2004). International cooperation in higher education: Theory and practice. Journal of Studies in International Education, 8(1), 32-55.

Charmaz, K. (2006). Constructing grounded theory: A practical guide through qualitative analysis. London, U.K.: Sage.

Dyer, J., Kale, P., \& Singh, H. (2001). How to make strategic alliances work. Sloan Management Review, 42(4), 37-43.

Easterby-Smith, M., Thorpe, R., \& Lowe, A. (2002). Management research: An introduction (2nd ed.). London, U.K.: Sage Publications Ltd..

Eisenhardt, K. M. (1989). Building theories from case study research. The Academy of Management Review, 14(4), 532-550.

Flyvbjerg, B. (2006). Five misunderstandings about case-study research. Qualitative Inquiry, 12(2), 219-245.

Glaser, B. G., \& Strauss, A. L. (1967). The discovery of grounded theory: Strategies for qualitative research. Chicago, I.L.: Aldine.

Gopal, K., \& Rabindra, N. B. (2014). Supply chain collaboration index: An instrument to measure the depth of collaboration. Benchmarking: An International Journal, 21(2), 184-204.

Gumport, P. J., \& Sporn, B. (1999). Institutional adaptation: Demands for management reform and university administration. In J. C. Smart, \& W. G. Tierney (Eds.), Higher education: Handbook of theory and research (Volume XIV, pp. 103-145). New York, N.Y.: Agathon Press.

Holden, M. T., \& Lynch, P. (2002). Choosing the appropriate methodology: Understanding research philosophy. Cork, Ireland: Waterford Institute of Technology. 
Hutchison, J., Johnston, L. H., \& Breckon, D. (2010). Using QSR-NVivo to facilitate the development of a grounded theory project: an account of a worked example. International Journal of Social Research Methodology, 13(4), 283-302.

Johnson, G., Scholes, K., \& Whittington, R. (2008). Exploring corporate strategy (8th ed.). Harlow, U.K.: Pearson Education Limited.

Janesick, J. R. (2001). Scientific charge-coupled devices. Bellingham, V.A.: SPIE Press.

Knox, K. (2004). A researcher's dilemma-Philosophical and methodological pluralism. Electronic Journal of Business Research Methods, 2(2), 119-128.

Kozlowski, S. W. J., Chao, G. T., Grand, J. A., Michael, T., Braun, M. T., \& Kuljanin, G. (2013). Advanced multilevel research design: Capturing the dynamics of emergence. Organizational Research Methods, 16(4), 581-615.

Li, X., Roberts, J., Yan, Y., \& Tan, H. (2014). Knowledge sharing in China-UK higher education alliance. International Business Review, 23(2), 343-355.

Nielsen, B. B. (2003). An empirical investigation of the drivers of international strategic alliance formation. European Management Journal, 21(3), 301-322.

Polkinhorne, D. E. (2005). Language and meaning: Data collection in qualitative research. Journal of Counselling Psychology, $52(2), 137-145$.

Robertson, A., Mullan, B., \& Todd, J. (2014). A qualitative exploration of experiences of overweight young and older adults. An application of the integrated behaviour model. Appetite, 75, 157-164.

Saunders, M., Lewis, P., \& Thornhill, A. (2012). Research methods for business students (6th ed.). Essex, U.K.: Pearson Education.

Sporn, B. (1999). Current issues and future priorities for European higher education systems. In P. G. Altbach, \& P. M. Peterson (Eds.), Higher education in the 21st century: Global challenge and national response (pp. 67-77). New York, N.Y.: Institute of International Education.

Stacy, M., Carter, S. M., \& Little, M. (2007). Justifying knowledge, justifying method, taking action: Epistemologies, methodologies, and methods in qualitative research. Qualitative Health Research, 17(10), 1316-1328.

Vauterin, J. J., Linnanen, L., \& Marttila, E. (2012). Value creation in international higher education: The role of boundary spanning in university-industry collaboration. International Journal of Quality and Service Sciences, 4(3), 283-298.

Zhang, Y., \& Wildemuth, B. M. (2009). Qualitative analysis of content. In B. Wildemuth (Ed.), Applications of social research methods to questions in information and library science (pp. 308-319). Westport, C.T.: Libraries Unlimited. 